\title{
New fatigue limit assessment approach of defective material under fully reversed tension and torsion loading
}

\author{
Hassine Wannes ${ }^{1, a}$, Anouar Nasr ${ }^{1,2}$ And Chokri Bouraoui ${ }^{1,3}$ \\ 1 LGM, ENIM, Avenue Ibn Eljazzar, 5019 Monastir, Tunisia \\ 2 EPEIM, Avenue Ibn Eljazzar, 5019 Monastir, Tunisia \\ 3 ENISO, Université de Sousse, BP 264, Cité Erriadh, 4023 Sousse, Tunisia
}

Received 14 April 2015, Accepted 27 August 2015

\begin{abstract}
This paper presents a new approach to predict the high cycle fatigue limit of defective material submitted to multiaxial loading. Defects are simplified to a half spherical void at the surface of a specimen. Finite Element (FE) method is used to determine stress distribution around defect for different sizes and loading levels. Papadopoulos high cycle fatigue criterion is used to calculate equivalent stress around defect. Based on stress analysis, a definition of affected area is proposed, in which the Papadopoulos criterion is violated. The evolution of the affected area, versus the amplitude of loading and defect size leads to determine fatigue limit for defective material. Results are in good agreement with experimental investigations and show that the affected area is a good parameter to predict the influence of a defect on multiaxial fatigue behaviour.
\end{abstract}

Key words: High cycle fatigue criterion / defect / fatigue limit / stress gradient / affected area

\section{Introduction}

The fatigue limit of components containing inherent defects is of great importance for industrial applications. Their lifespan is always depending on morphology of the defect and the loading path. Due to the production process various types of casting defects can affect mechanical parts such as: inclusion, shrinkage, and gas pore or dross defect [1]. The designer needs to create balance by taking into account the real effect of the size of defect on the fatigue limit of the component to correlate the fatigue limit with defect size. The aim of this paper is to propose a new approach that allows to predict the HCF limit of a mechanical part containing defect.

Several methods are explored and different authors have proposed fatigue limit calculation corresponding to different sizes of defect. Murakami [2] proposed an interesting approach based on experimental results. A geometrical parameter, $\sqrt{\text { area }}$, defined as the square root of the projected area of defect on the plane perpendicular to the direction of the maximal principal stress, is used to identify the defect size. This approach suggests a practical relationship between the fatigue limit, and this proposed parameter. This model is limited to the uniaxial fatigue cases and gives conservative results especially in the case of torsion loading.

\footnotetext{
${ }^{a}$ Corresponding author: hassine.wannes@gmail.com
}

Endo [3] has extended Murakami's criterion to proportional biaxial loading, where the ratio between torsion and tension fatigue limits is supposed to be constant whatever the size of the defect. However fatigue limits under combined loading are deduced without a fatigue test. Murakami [4] considers the defect as a crack where the size is characterized by the $\sqrt{a r e a}$ parameter. He considers that crack initiation stage is negligible. Fatigue life is therefore assumed to be controlled by the crack propagation law.

Other authors consider the defect as equivalent to a notch [5]. The fatigue limit will be linked to defect size by taking into account the type of defect by means of the elastic stress concentration factor usually named $K_{t}$. The defect is modelled as a half spherical hole at the surface of specimen with $K_{t}=2$. Such an approach provides good results for macroscopic notches but it is limited to uniaxial loading.

Many authors tend to determine the fatigue limit under different assumptions such as the critical distances method proposed by Taylor [6] which is applied on cracks or notches. He used the linear elastic fracture mechanics under tensile stress modified by an introduction of material parameter constant.

Nadot et al. [7] studied FGS 52 containing natural defects in surface and on the bulk, the authors conclude that for a given size, surface defects are much more harmful 


\section{Nomenclature}

\begin{tabular}{|c|c|}
\hline Affected area & Area where Papadoupolos criterion was violated $\left[\mu \mathrm{m}^{2}\right]$ \\
\hline Affected arealimt & Affected area at fatigue limit $\left[\mu \mathrm{m}^{2}\right]$ \\
\hline$E$ & Young's modulus [MPa] \\
\hline HLP & Highest Loaded Plane \\
\hline$k_{w 1}$ & Material parameter used in case of tension loading \\
\hline$k_{w 2}$ & Material parameter used in case of torsion loading \\
\hline$R$ & Radius of defect, $[\mu \mathrm{m}]$ \\
\hline$r$ & Distance from the centre of defect to a considered point on HLP $[\mu \mathrm{m}]$ \\
\hline$R_{m}$ & Tensile strength $[\mathrm{MPa}]$ \\
\hline$R_{p 0.2}$ & Yield strength at $0.2 \%$ plastic deformation $[\mathrm{MPa}]$ \\
\hline$R_{p 0.2 c y}$ & Cyclic yield stress $[\mathrm{MPa}]$ \\
\hline$R_{\sigma}$ & Load ratio between minimum and maximum stress of the loading cycle \\
\hline$T_{a}$ & Generalized amplitude of shear stress for each plane $\Delta[\mathrm{MPa}]$ \\
\hline \multicolumn{2}{|r|}{ Greek letters } \\
\hline$\alpha$ & Material parameter in the Papadoupolos equivalent stress \\
\hline$\beta$ & Material parameter in Papadoupolos criterion [MPa] \\
\hline$\varepsilon_{p l}$ & Plastic strain \\
\hline$\theta$ & Angle describing the location of plane $\Delta$ \\
\hline$\nu$ & Poisson's ratio \\
\hline$\sigma_{a}$ & Amplitude of the load cycle under tension loading [MPa] \\
\hline$\sigma_{D}$ & Fatigue limit under tension loading of defective material $[\mathrm{MPa}]$ \\
\hline$\sigma_{D-1}$ & Fully reversed tension fatigue limit of defect free material [MPa] \\
\hline$\sigma_{\text {eqpap }}$ & Papadoupolos equivalent stress $[\mathrm{MPa}]$ \\
\hline$\tau_{a}$ & Amplitude of the load cycle under torsion loading [MPa] \\
\hline$\tau_{D}$ & Fatigue limit under torsion loading of defective material $[\mathrm{MPa}]$ \\
\hline$\tau_{D-1}$ & Fully reversed torsion fatigue limit of defect free material $[\mathrm{MPa}]$ \\
\hline$\tau_{\theta, \varphi}$ & Vector of shear stress, defined in $\Delta[\mathrm{MPa}]$ \\
\hline$\varphi$ & Angle describing the location of plane $\Delta$ \\
\hline$\Delta$ & Plane crossing the calculate point of Papadoupolos criterion \\
\hline$\sqrt{\text { area }}$ & Murakami parameter describing defect size $[\mu \mathrm{m}]$ \\
\hline
\end{tabular}

than those located in the bulk. They conclude that the severity of the defect affects the fatigue limit.

By their experimental results Billaudeau et al. [8] show that for both, tension and torsion loading, the use of a fatigue criterion made for notches or cracks does not enable to predict an accurate defect fatigue behaviour. They conclude that for a given size, the shape of the defect modifies the fatigue limit under tension and torsion loading when the stress concentration factor is lower than $2\left(K_{t}<2\right)$. For $K_{t}>2$ the fatigue limit is independent of the defect geometry. Finally they show that the ratio between torsion and tension fatigue limits, which is supposed to be constant, depends on defect geometry and balances from 0.72 to 1 .

Recently, some authors [9-11] observe that fatigue crack initiates at the tip of the surface defect within the maximum shear plane and crack was observed to propagate in the plane which is perpendicular to the direction of the maximal principal stress. Such observations were confirmed by FE analysis describing the stress distribution around the defect. This FE analysis shows the influence of the gradient of the maximal hydro-static stress, which is taken into account on the HCF criterion for defective material. This approach has the advantage to be applicable in different load ratios. This methodology, called Defect Stress Gradient (DSG), provides good results in cases of spherical pore defects subjected to fully reversed loading for $R_{\sigma}=-1$. However for $R_{\sigma}=0.1$ results are not as good as those corresponding to $R_{\sigma}=-1$ [12]. In the case of elliptical defects DSG criterion underestimates the fatigue limit [13] and confirms that the only and simple parameter size $=\sqrt{a r e a}$ is not able to describe the ellipsoidal defect influence.

In the present paper we propose a new approach to correlate fatigue limit to defect size where triaxiality of stress gradient around defect is taken into account. Based on FE analysis, an affected area is defined, which is the area close to the defect from which the Papadoupolos fatigue criterion is violated.

Papadoupolos equivalent stress is interpolated on the plane perpendicular to the maximum principal stress. This interpolation will be used to calculate the affected area. The evolution of the affected area versus the loading amplitude and defect size is used to determine the fatigue limit for each defect size.

The proposed approach is applied for two different materials submitted to fully reversed tension and torsion loading. Results are in good agreement with experimental investigations and results are improved comparing to those given by other approaches presented in the literature. The proposed approach shows that the affected area is a good parameter to characterize the influence of a defect on the fatigue behaviour. 


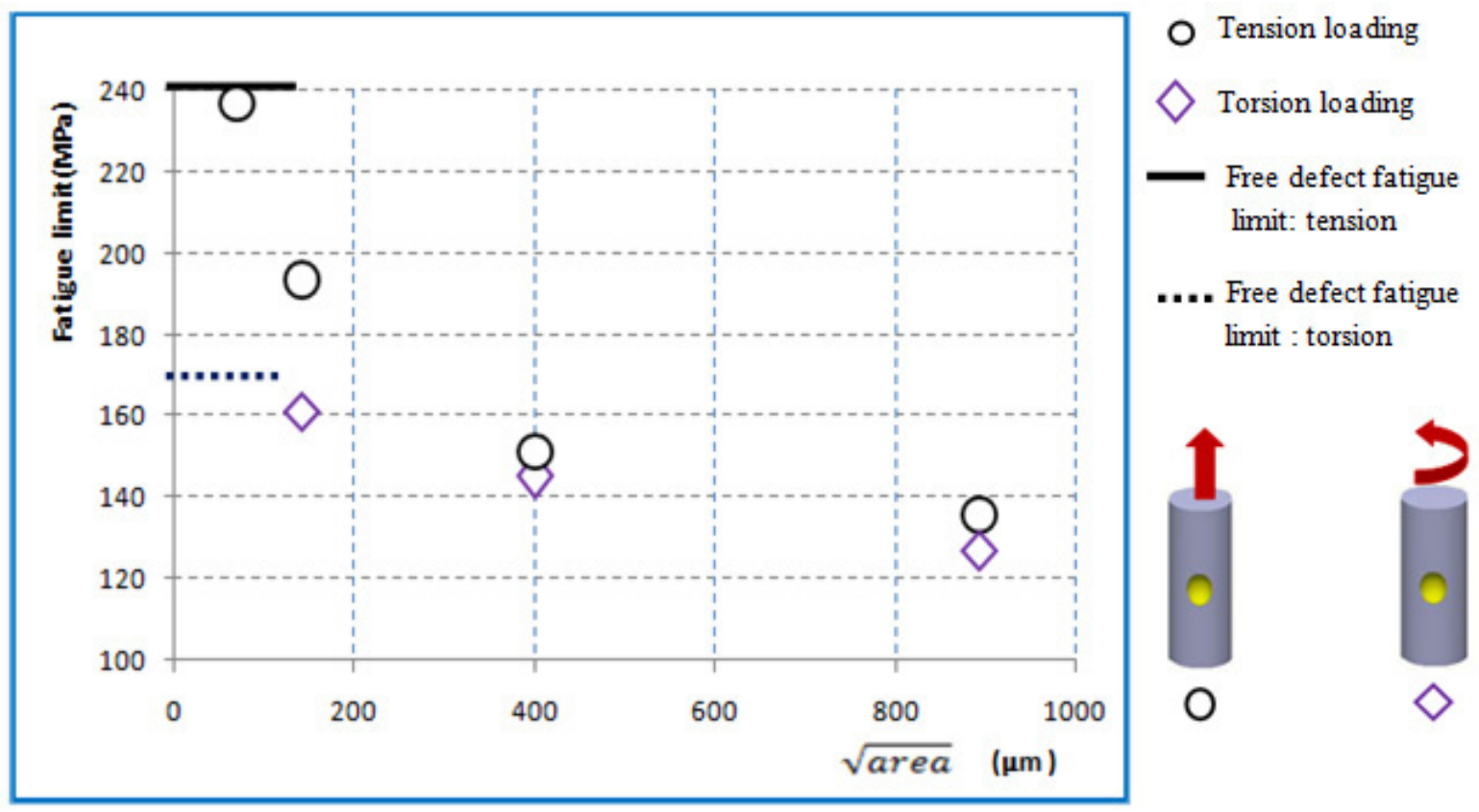

Fig. 1. Experimental evolution of fatigue limit with defect size $(\sqrt{\text { area }})$ [12].

It is important to note that the model has the advantage to be simple and does not need many parameters to identify. In addition, it can be applied to loading with mean stress and it can be extended to multiaxial loadings since an analytical solution could be determined.

\section{Material and experimental database}

This study is carried out on the 1045 steel used in aircraft and automotive industry. The main mechanical properties are: Young modulus $E=195$ GPa, Poisson's ratio $\nu=0.3$, the $0.2 \%$ offset yield stress $R_{p 0.2 \%}=$ $359 \mathrm{MPa}$, the cyclic yield limit $R_{p c 0.2 \%}=250 \mathrm{MPa}$, the ultimate tensile strength $R_{m}=594 \mathrm{MPa}$ and the fracture lengthening $A=31 \%$. An experimental database containing multiaxial fatigue results for 1045 steel with induced defects has been used [13].

Massive standard cylindrical specimens, with $5 \mathrm{~mm}$ and $12 \mathrm{~mm}$ diameters are used respectively for tension and torsion fatigue tests. Artificial spherical surface defects with controlled size have been machined, at the middle length of fatigue sample, by using electric discharge machining (EDM) process [8]. Due to residual stress induced by the process a tempering is done after every introduction $\left(500{ }^{\circ} \mathrm{C} / \mathrm{h}\right.$ under vacuum) to minimize the residual stress effect.

The tension and torsion fatigue tests were carried out for fully reversed loading (load ratio $R_{\sigma}=-1$ ) at respectively 100 and $45 \mathrm{~Hz}$. The samples are subjected to a cycle number greater than $10^{6}$ cycles. Figure 1 shows fatigue limit evolution with $\sqrt{\text { area }}$ for fully reversed tension and torsion loading for spherical pore on surface.
These experimental results in Figure 1 show that the fatigue limit of a specimen containing a defect is sensitive to the defect size and to the loading path.

\section{Papadoupolos criterion}

In this study the last formulation of Papadoupolos $\mathrm{HCF}$ criterion is used [14]. It can be defined as follows: in a material volume $V$, a plane $\Delta$ is defined by its normal, $\vec{n}$, this vector makes an angle $\theta$ with $z$-axis of the $O_{x y z}$ frame as shown in Figure 2.

The projection of this vector on the $x y$ plane makes an angle $\varphi$ with the $x$-axis.

Papadoupolos et al. [13] introduce a so called resolved shear stress $\tau(t)$, which is the projection of the shear vector $\vec{C}(t)$ on a straight line $\zeta=\vec{m}(\psi)$ of $\Delta$ plane.

$$
\tau(t):=\vec{C}(t) \cdot \vec{m}
$$

The amplitude of the resolved shear stress $\tau_{a}$ acting on $\Delta$ along $\zeta$ for $t \in P$ is:

$$
\tau_{a}(\theta, \varphi, \psi)=\frac{1}{2}\left[\max _{t} \tau(\varphi, \theta, \psi, t)-\min _{t} \tau(\varphi, \theta, \psi, t)\right]
$$

$P$ is the cycle loading period.

Finally, for a given plane $\Delta$, and for a fixed couple $(\theta, \varphi)$, the generalized shear stress amplitude $T_{a}$ is defined as:

$$
T_{a}(\theta, \varphi)=\sqrt{\frac{1}{\pi} \int_{=0}^{2 \pi} \tau_{a}^{2}(\theta, \varphi, \psi) d \psi}
$$

The Papadoupolos criterion is then defined as:

$$
\sigma_{\text {eqpap }}=\theta \max _{\theta, \varphi}\left(T_{a}\right)+\alpha \sigma_{H, \max } \leqslant \beta
$$



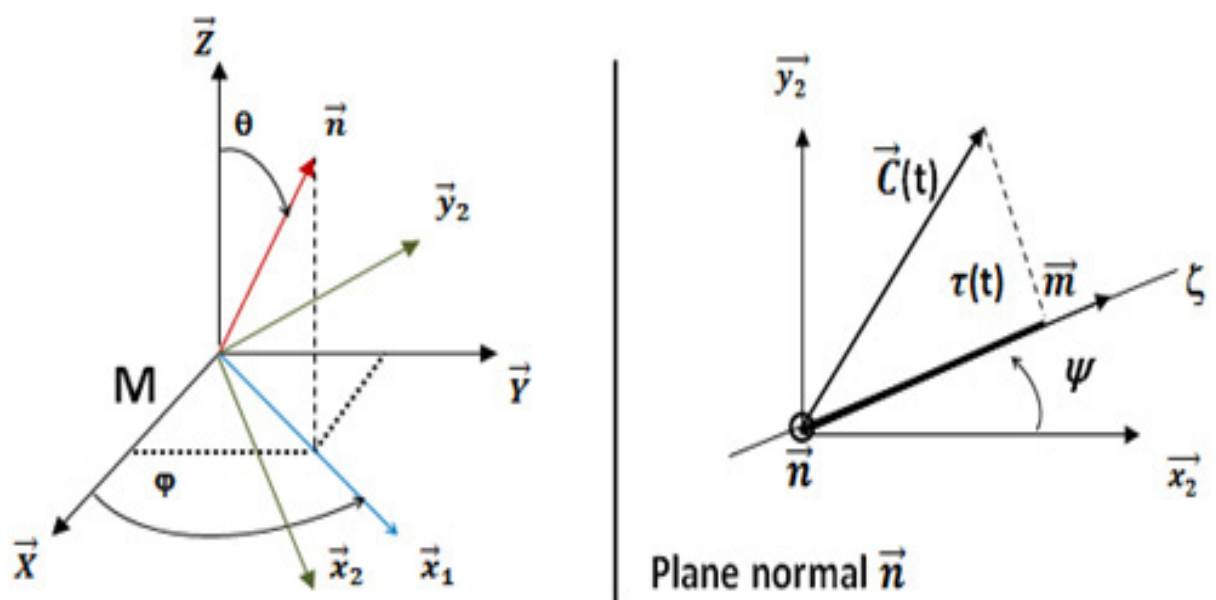

\section{Plane normal $\vec{n}$}

Fig. 2. Definition of terms related to shear stress [13].

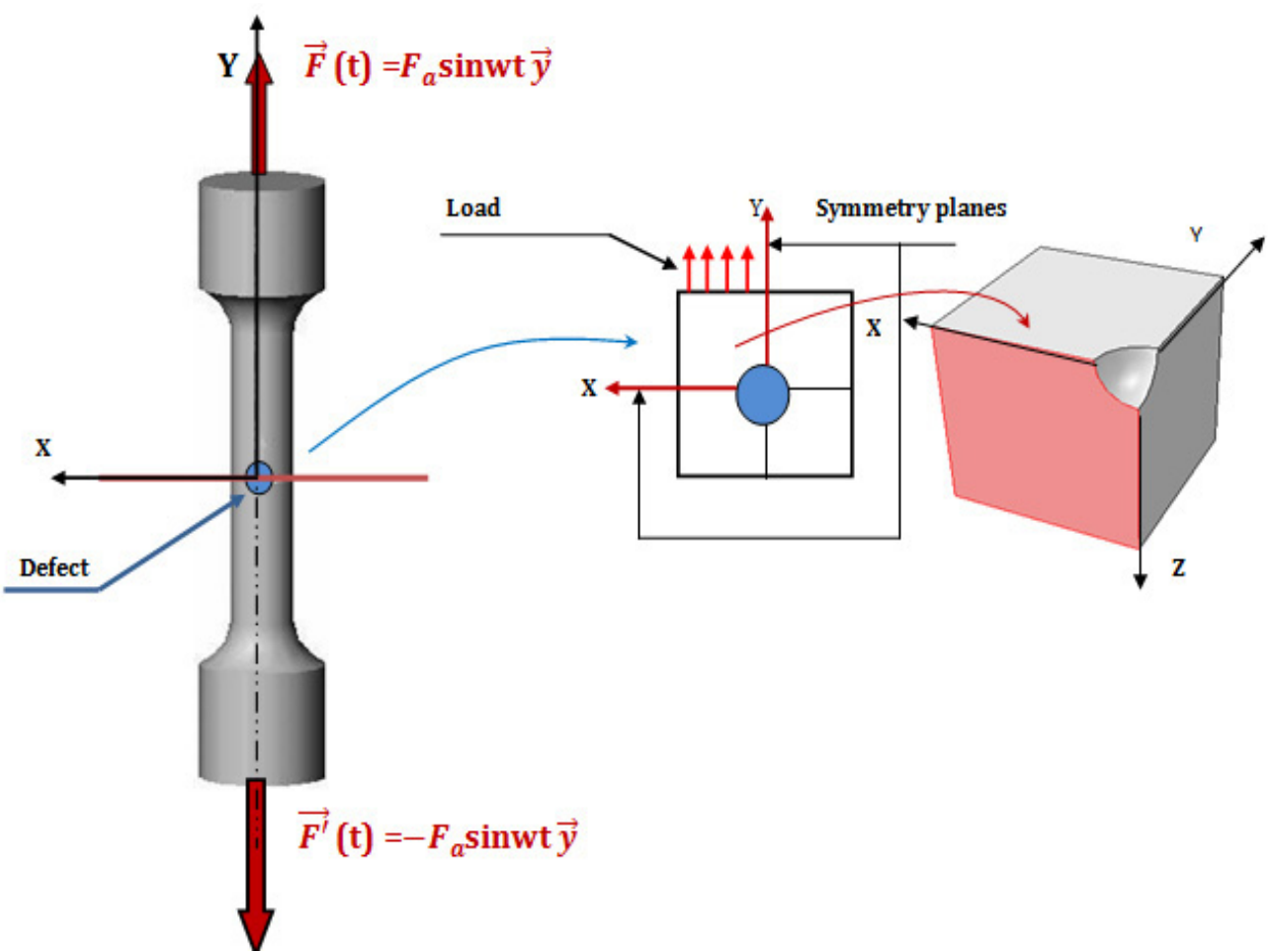

Fig. 3. Fully completed model and simplified model.

The quantity $\sigma_{H \text {,max }}$ is the maximum value of the hydrostatic stress within a loading cycle.

As long as this relationship is satisfied, there is no initiation cracking risk. Both $\alpha$ and $\beta$ parameters are function of the material fatigue properties such as:

$$
\beta=\tau_{D-1} \quad \text { and } \quad \alpha=3\left(\frac{\tau_{D-1}}{\sigma_{D-1}}-\frac{1}{2}\right)
$$

where $\tau_{D-1}$ and $\sigma_{D-1}$ are the fully reversed torsion and tension fatigue limits, respectively.

\section{The 3D finite element simulation of stress gradient around the defect}

\subsection{Finite element modelling}

It is worth noticing that defects are generally the preferential sites for crack initiation. It is in great interest to characterize the stress distribution around the defect. Due to the symmetry of the problem (geometry and loading), only one fourth parallelepiped is considered for the FE simulations as shown in Figure 3. Symmetry and boundary conditions are applied as shown in Figure 4. The geometry is meshed by means of four-node linear tetrahedral 

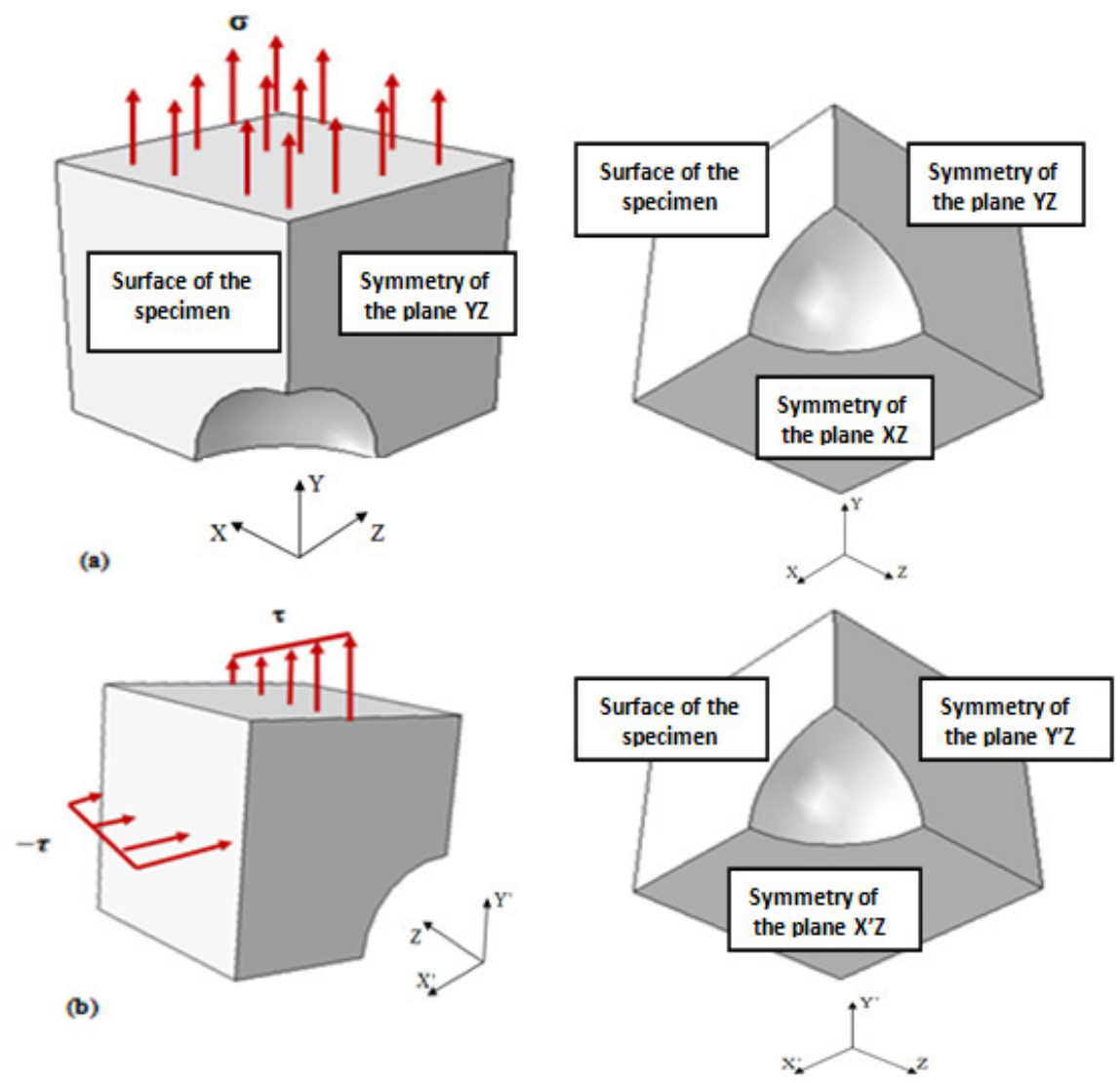

Fig. 4. Load and boundary conditions applied in the FE model: (a) tension loading, (b) torsion loading.

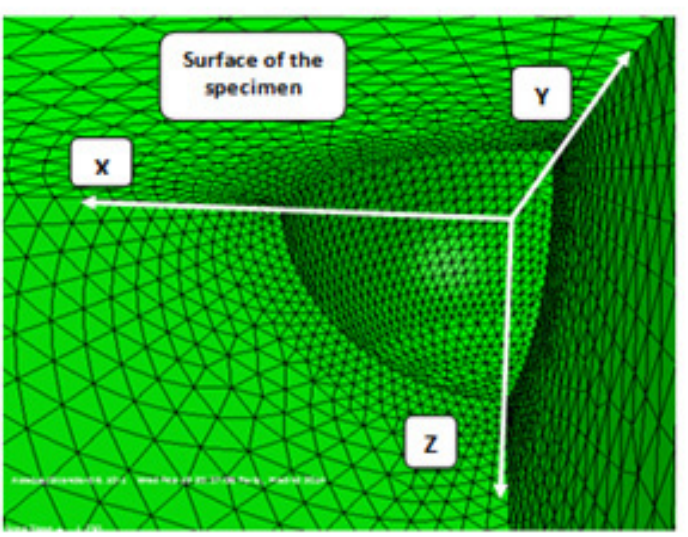

(a)

$$
\sigma_{a}(M P a)
$$

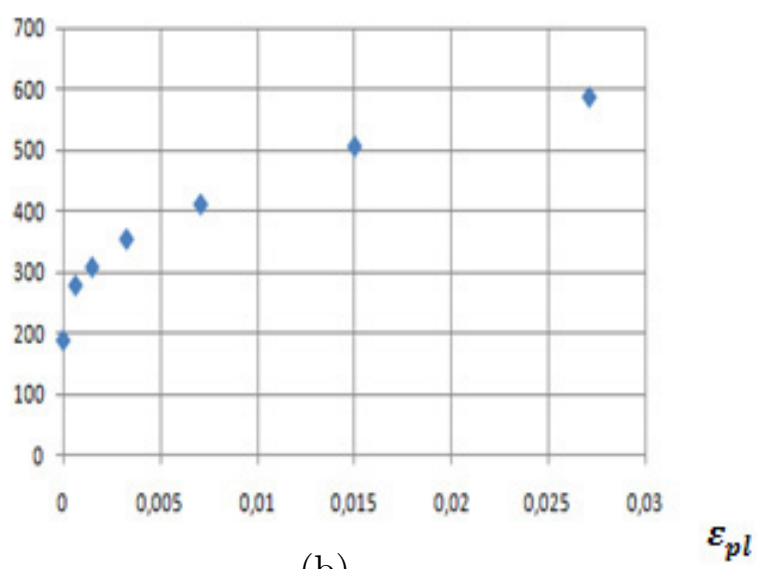

(b)

Fig. 5. (a) Refined mesh around defect. (b) Plastic stress-strain cyclic stabilized experimental curve.

solid elements C3D4. Meshing is refined and optimized in the critical zone around the defect (Fig. 5a).

Since plasticity occurs around the defect all simulations are conducted with the elastic-plastic Prandtl-Reuss behaviour type model based on Von Mises energetic criterion with a linear isotropic hardening law. The cyclic stabilized experimental stress-strain curve used to identify the model is shown on Figure 5b.

\subsection{Stress distribution around defect}

Based on FE calculation, the highest loaded plane (HLP) is the plane which is perpendicular to the maximum stress direction (Fig. 6). In addition the experimental works provide that crack initiation occurs always at the tip of the defect in the shear plane [8]. The macroscopic crack that leads to the specimen failure grows in 


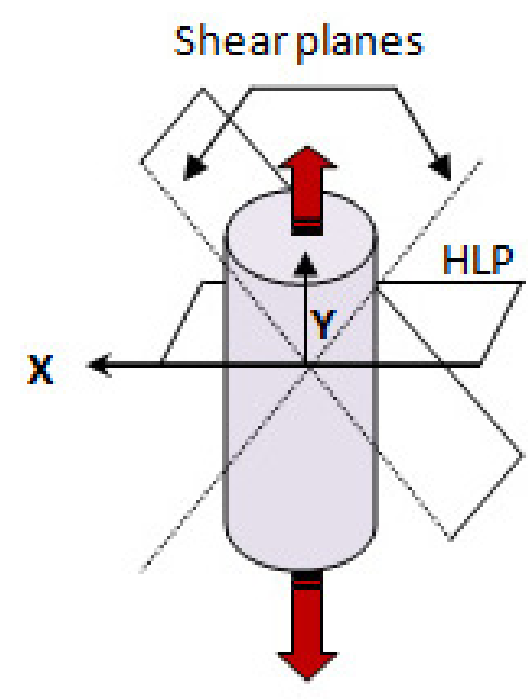

(a)

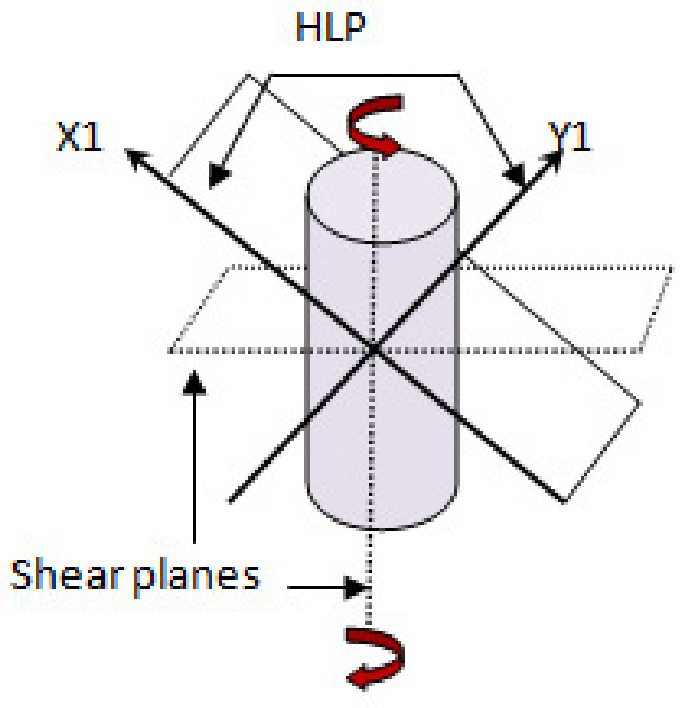

(b)

Fig. 6. Shear planes and highest load planes: (a) tension loading, (b) torsion loading.

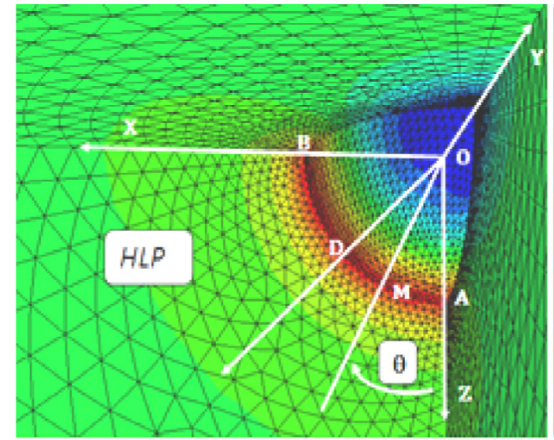

(a)

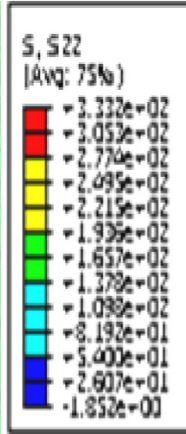

$1.852=01$

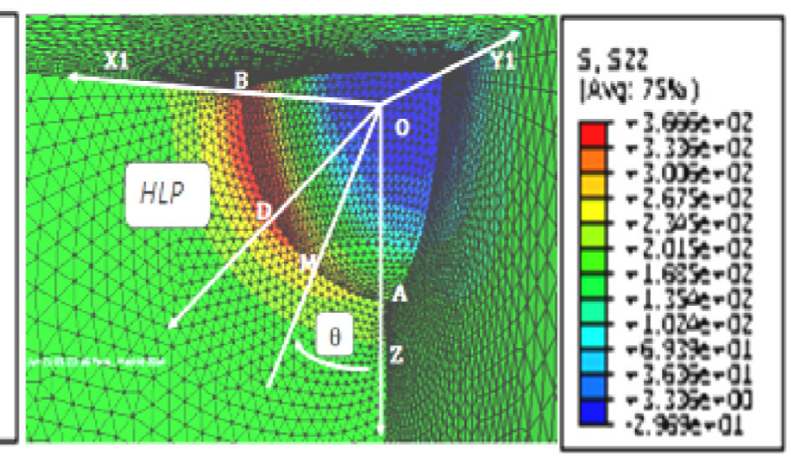

(b)

Fig. 7. Stress distribution around defect: S22 (Pas). (a) Tension loading $\sigma_{a}=180$ MPa. (b) Torsion loading $\tau_{a}=150 \mathrm{MPa}$.

the HLP. Consequently we will be interested in the distribution of stress on this plane (Fig. 7).

\subsection{Analysis of FE results}

\subsubsection{Tension results}

Numerical calculations of $\sigma_{\text {eqpap }}$ in HLP show that:

- $\sigma_{\text {eqpap }}$ is almost constant along the defect radius (AB) with a scattering that does not exceed $3 \%$ (Fig. 8).

- In HLP the defect gradient of $\sigma_{\text {eqpap }}$ in any direction starting from the defect centre is quite similar (Fig. 9).

In HLP the distribution of Papadoupolos equivalent stress is independent of the chosen direction. It depends only on the ratio $r / R$ and the applied load. Consequently in HPL $\sigma_{\text {eqpap }}$ can be interpolated by the following equation:

$$
\sigma_{\text {eqpap }}(r)=\frac{\tau_{D-1}}{\sigma_{D-1}} \sigma_{a}\left(\frac{1}{(r / R)^{4}}+1\right)
$$

where:

$\sigma_{D-1}$ : Defect free fatigue limit under fully reversed tension loading.

$\tau_{D-1}$ : Defect free fatigue limit under fully reversed torsion loading.

$\sigma_{a}: \quad$ Amplitude of applied loading.

$R: \quad$ Defect radius.

$r$ : Distance from defect centre to the considered point.

\subsubsection{Torsion results}

Numerical calculation of $\sigma_{\text {eqpap }}$ in HLP show that:

- $\sigma_{\text {eqpap }}$ is almost constant along the defect radius (AB) with a scattering that does not exceed $9 \%$ (Fig. 10).

- In HLP close to defect the defect gradient of $\sigma_{\text {eqpap }}$ in any direction starting from the defect centre is quite similar (Fig. 11). 


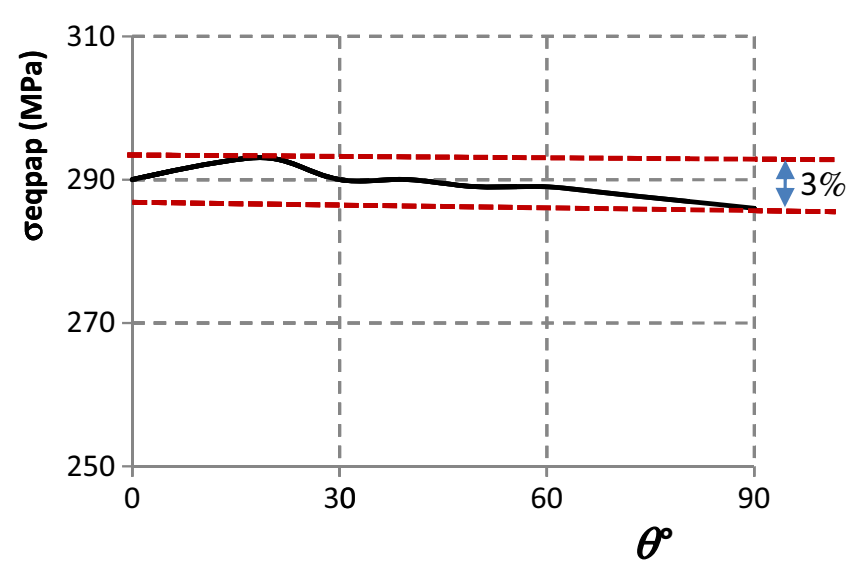

Fig. 8. Variation of $\sigma_{\text {eqpap }}$ along the defect radius (AB) $\left(\sqrt{\text { area }}=300 \mu \mathrm{m}, \sigma_{a}=210 \mathrm{MPa}\right)$.

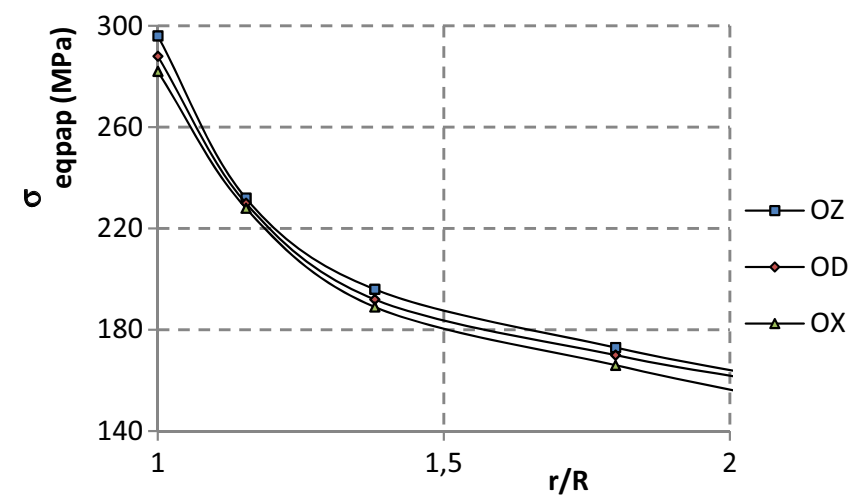

Fig. 9. Variation of $\sigma_{\text {eqpap }}$ along three directions on HLP $\left(\sqrt{\text { area }}=300 \mu \mathrm{m} ; \sigma_{a}=210 \mathrm{MPa}\right)$.

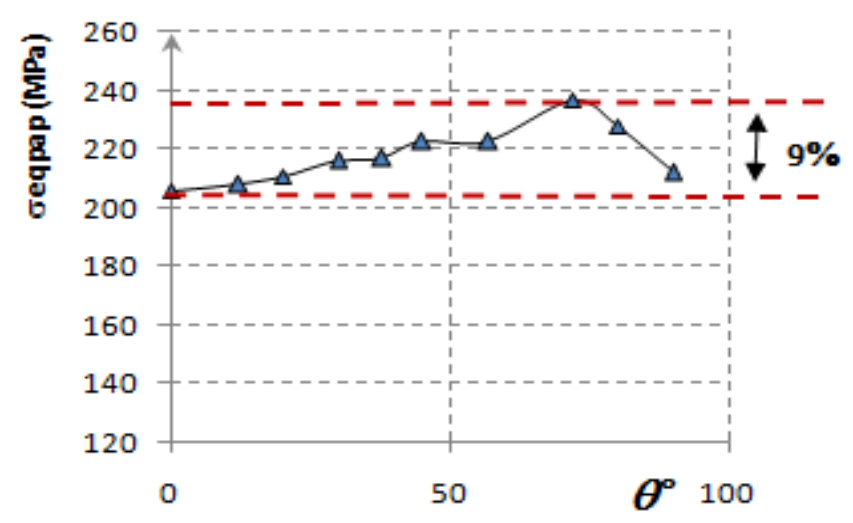

Fig. 10. Variation of $\sigma_{\text {eqpap }}$ along the defect radius (AB) $\left(\sqrt{\text { area }}=300 \mu \mathrm{m} ; \tau_{a}=150 \mathrm{MPa}\right)$.

In HLP close to defect the distribution of Papadoupolos equivalent stress is independent of the chosen direction. It depends only on the ratio $r / R$ and the applied load. Consequently in HPL $\sigma_{\text {eqpap }}$ can be interpolated by the following equation:

$$
\sigma_{\text {eqpap }}(r)=\frac{\tau_{D-1}}{\sigma_{D-1}} \tau_{a}\left(\frac{1}{(r / R)^{2}}+1\right)
$$

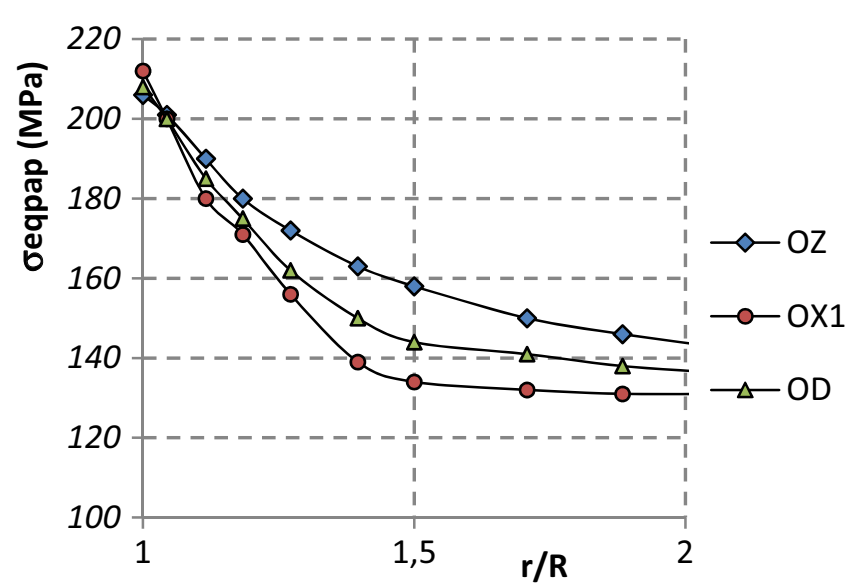

Fig. 11. Variation of $\sigma_{\text {eqpap }}$ along three directions on HLP $\left(\sqrt{\text { area }}=300 \mu \mathrm{m} ; \tau_{a}=150 \mathrm{MPa}\right)$.

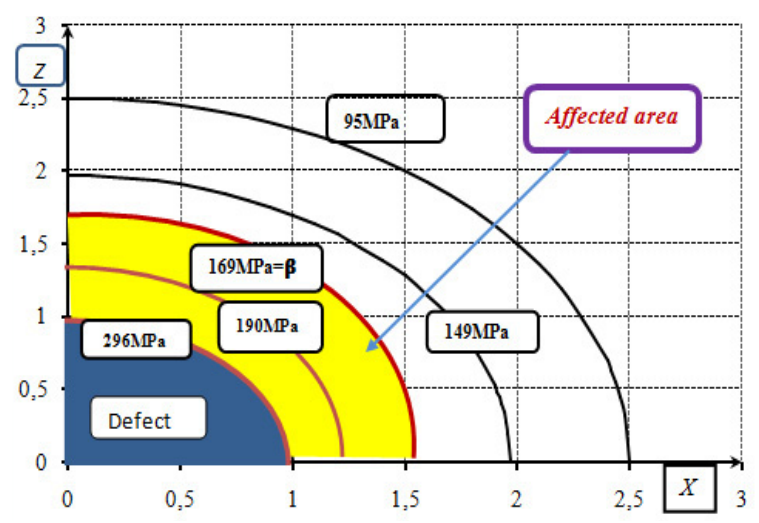

Fig. 12. $\sigma_{\text {eqpap }}$ distribution and affected area in $\operatorname{HLP}\left(\sigma_{a}=\right.$ $210 \mathrm{MPa} ; \sqrt{\text { area }}=300 \mu \mathrm{m})$.

where:

$$
\tau_{a} \text { : Amplitude of applied loading. }
$$

Equations (6) and (7), used to interpolate $\sigma_{\text {eqpap }}$ on HLP are validated by a numerical simulation. They lead to a good results with a difference less than $5 \%$.

\section{New HCF limit assessment approch of defective material}

\subsection{Methodology}

From the results of numerical simulations in HLP it is shown that the variation of $\sigma_{\text {eqpap }}$ is independent of the chosen direction. $\sigma_{\text {eqpap }}$ distribution in HLP shows iso-value circular arches centred around defect (Fig. 12).

In HLP there is an area, close to the defect, from which the Papadoupolos criterion is violated. Consequently the affected area for a given defect and an applied load is defined as the area where the Papadoupolos equivalent stress is greater than or equal to $\beta$. 


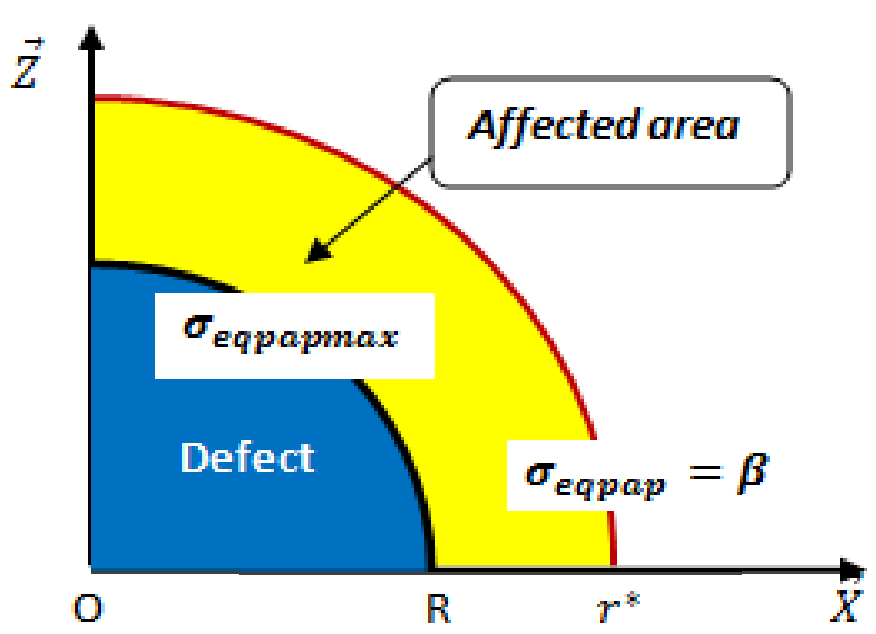

Fig. 13. Affected area calculation.

The affected area depends on applied loading and defect size. For a given defect size, under fatigue limit loading, it corresponds an affected area limit. If the affected area is less than affected area limit, there is no failure before $10^{7}$ cycles.

At fatigue limit loading the affected area is equal to the affected area limit.

Using Equations (6) and (7) we can determine the affected area as defined above in different cases corresponding to different loading modes.

\subsubsection{Fully reversed tension loading}

The affected area is determined using $R$ and $r^{*}$ (Fig. 13). From Equation (6) $r^{*}$ is deduced as follow:

$$
\beta=\frac{\tau_{D-1}}{\sigma_{D-1}} \sigma_{a}\left(\frac{1}{\left(r^{*} / R\right)^{4}}+1\right)
$$

when $\beta$ is equal to $\tau_{D-1}$, then:

$$
r^{*}=\left(\frac{1}{\frac{\sigma_{D-1}}{\sigma_{a}}-1}\right)^{0.25} R
$$

$$
\begin{aligned}
\text { Affected area } & =\frac{\pi}{4}\left(r^{* 2}-R^{2}\right) \\
\text { and } \sqrt{\text { area }} & =\sqrt{\frac{\pi R^{2}}{2}}
\end{aligned}
$$

From Equations (10) and (11) the affected area is assessed as follow:

$$
\begin{aligned}
\text { Affected area } & =\frac{1}{2}\left(K^{2}-1\right)(\sqrt{\text { area }})^{2} \\
\text { where } K & =\left(\frac{1}{\frac{\sigma_{D-1}}{\sigma_{a}}-1}\right)^{0.25}
\end{aligned}
$$

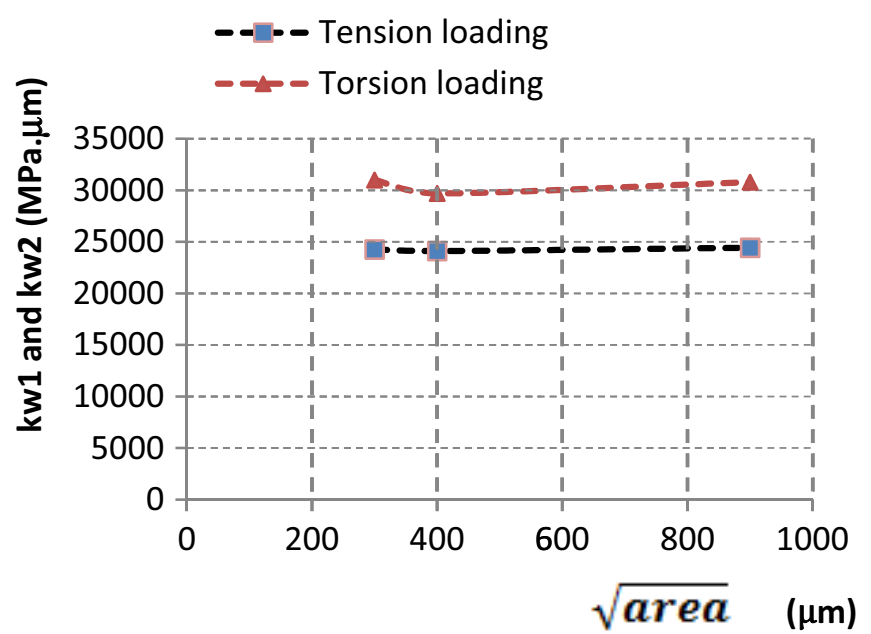

Fig. 14. $k_{w 1}$ and $k_{w 2}$ versus the defect size $(\sqrt{a r e a})$.

\subsubsection{Fully reversed torsion loading}

Using Equation (7) the affected area in case of reversed torsion can be determinate by:

$$
\begin{aligned}
\text { Affected area } & =\frac{1}{2}\left(H^{2}-1\right)(\sqrt{\text { area }})^{2} \\
\text { where } H & =\left(\frac{1}{\sigma_{D-1} / \tau_{a}-1}\right)^{0.5}
\end{aligned}
$$

From the experimental data base (Table 1), the affected area limit is determined by Equations (12) or (14), at the fatigue limit loading values in each case.

Now we denote:

$$
\sigma_{D} \sqrt{\text { Affected area }}_{\text {limit }}=k_{w 1}
$$

in case of reversed fully tension loading

$$
\tau_{D} \sqrt{\text { Affected area }}_{\text {limit }}=k_{w 2}
$$

in case of fully reversed torsion loading.

Figure 14 shows $k_{w 1}$ and $k_{w 2}$ versus defect size for the two loading modes. The main deduction is that $k_{w 1}$ and $k_{w 2}$ are almost constant and independent of the defect size.

\subsubsection{Proposed approach}

Based on experimental results in Table 1 for fully reversed tension and torsion loading the product of fatigue limit of defective material by the square root of affected area $a_{\text {limit }}$ is almost constant and independent of defect size.

The proposed approach is summarized on the following steps:

(i) From an experimental fatigue limit of medium defect size using Equations (16) or (17) $k_{w 1}$ or $k_{w 2}$ are deduced. 
Table 1. Experimental results $[7,12]$.

\begin{tabular}{cccc}
\hline \multicolumn{4}{c}{ Fully reversed tension loading } \\
\hline $\begin{array}{c}\text { Defect size } \sqrt{\text { area }} \\
(\mu \mathrm{m})\end{array}$ & $\begin{array}{c}\sigma_{D} \\
(\mathrm{MPa})\end{array}$ & $\begin{array}{c}\text { Affected area } \\
\left(\mu \mathrm{m}^{2}\right)\end{array}$ & $\begin{array}{c}k_{w 1} \\
(\mathrm{MPa} . \mu \mathrm{m})\end{array}$ \\
\hline 170 & 195 & 15630 & 24378 \\
400 & 152 & 25140 & 24100 \\
900 & 130 & 35280 & 24418 \\
\hline \multicolumn{4}{c}{ Fully reversed torsion loading } \\
\hline Defect size $\sqrt{\text { area }}$ & $\tau_{D}$ & Affected area & \\
$(\mu \mathrm{m})$ & $(\mathrm{MPa})$ & $\left(\mu \mathrm{m}^{2}\right)$ & $(\mathrm{MPa} . \mu \mathrm{m})$ \\
\hline 300 & 157 & 20642 & 31000 \\
400 & 145 & 42105 & 29700 \\
900 & 128 & 57857 & 30788 \\
\hline \multicolumn{4}{c}{}
\end{tabular}

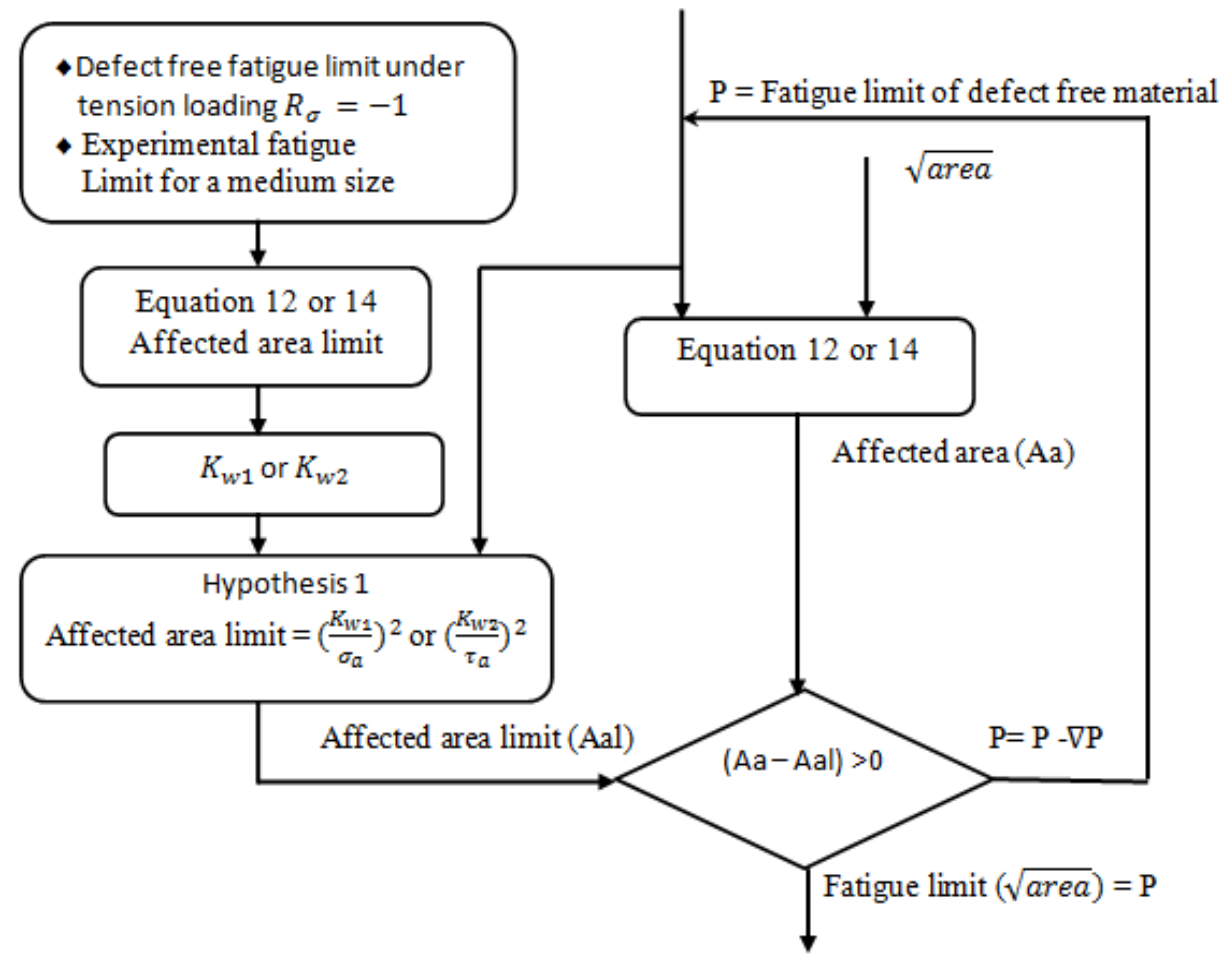

Fig. 15. Flowchart to simulate Kitagawa diagram.

(ii) On the same graph the following curves are plotted:

- Affected area versus loading amplitude for several defect sizes Equations (2) or (14)

- Affected area limit versus loading amplitude:

$$
\text { Affected area } \text { limit }=\left(\frac{k_{w 1}}{\sigma_{a}}\right)^{2} \text { or }\left(\frac{k_{w 2}}{\tau_{a}}\right)^{2}
$$

(iii) Finally the crossing points allow to determine the fatigue limit for each defect size. The necessary steps for the proposed approach are summarized in the flowchart (Fig. 15). With a simple Matlab program fatigue limit value is determined for each defect size in both cases: fully reversed tension or torsion loading and for different materials.

\subsection{Applications}

\subsubsection{Alternative tension loading}

In alternative tension loading case the affected area is calculated using Equation (12). From an experimental fatigue limit of a medium defect size the parameter $k_{w 1}$ is determined. In this study, a defect size $\sqrt{\text { area }}=400 \mu \mathrm{m}$ corresponding to a fatigue strength $\sigma_{D}=152 \mathrm{MPa}$ is used.

Using the proposed approach we deduce that $k_{w 1}=$ $24100 \mathrm{MPa} . \mu \mathrm{m}$.

In a same graph (Fig. 16) the following curves are plotted:

- Affected area versus loading amplitude for many defect sizes Equation (12). 


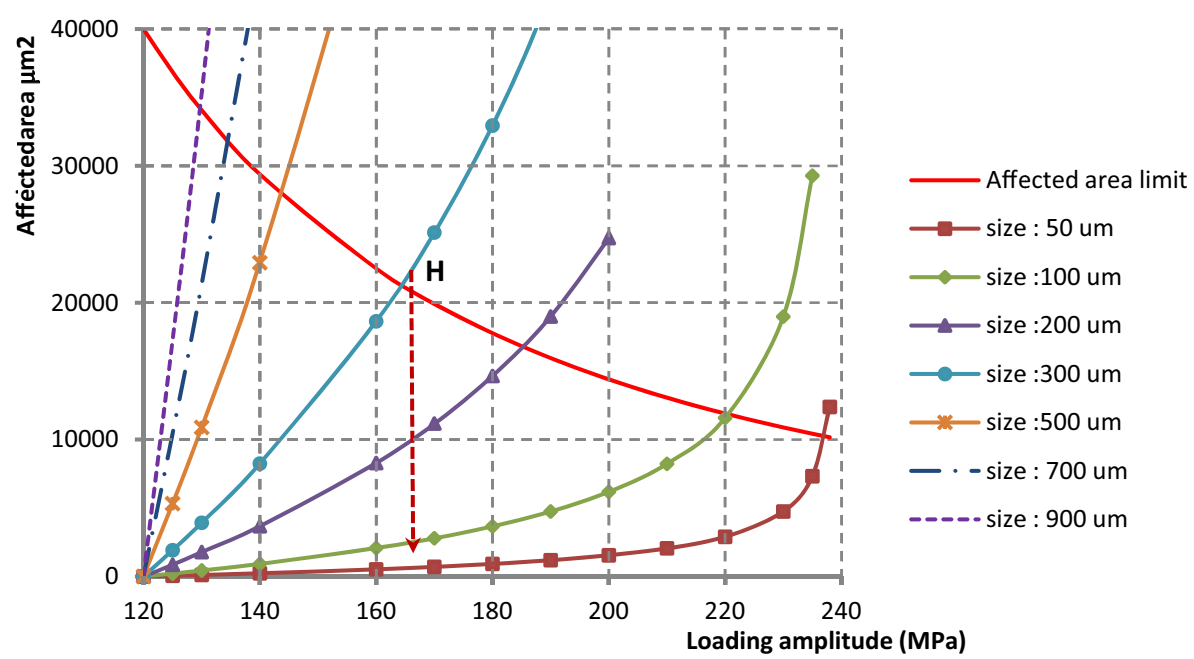

Fig. 16. Application of proposed approach: fully reversed tension (Material 1045 steel).

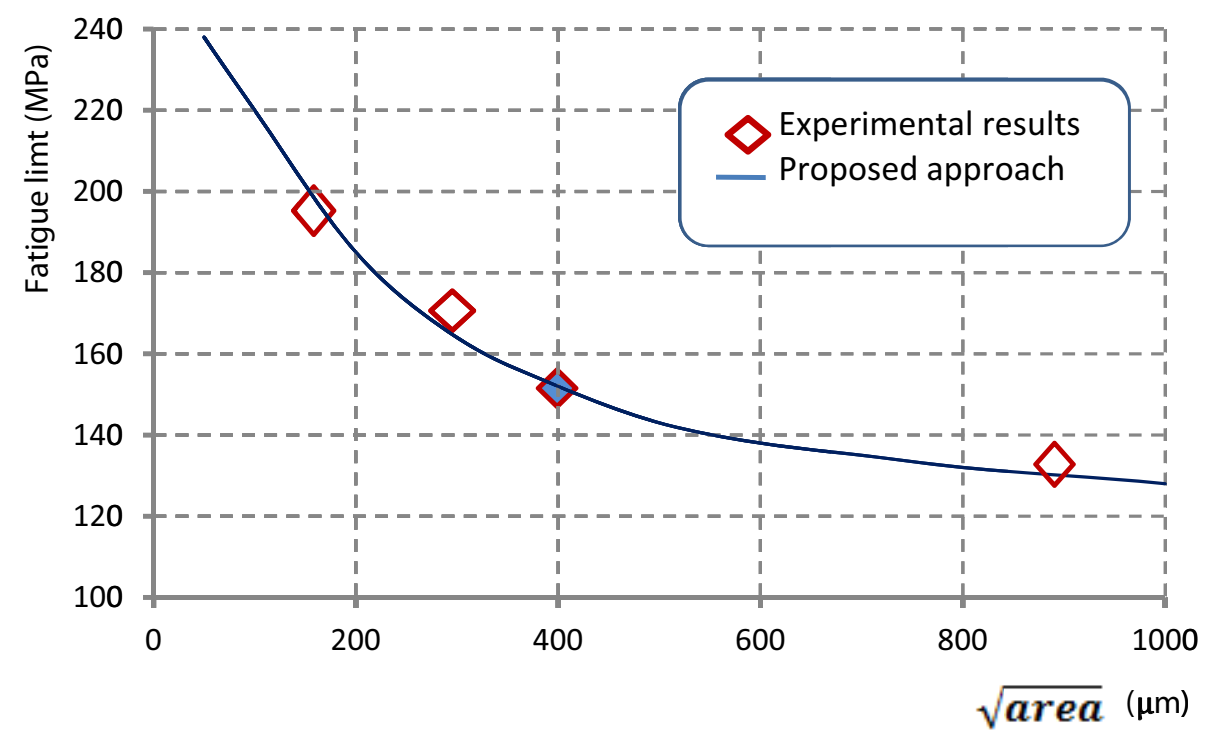

Fig. 17. Kitagawa diagram tension loading $R_{\sigma}=-1$ (Material 1045 steel).

- Affected arealimit versus loading amplitude Equation (18).

From the crossing point $\mathrm{H}$ a defect size $\sqrt{\text { area }}=300 \mu \mathrm{m}$ corresponding to the fatigue limit $\sigma_{D} \approx 165 \mathrm{MPa}$ is deduced. Comparing to experimental results a good concordance is noted (Fig. 17).

Using all crossing points, for each defect size Kitagawa diagram can be deduced in the case of fully reversed tension loading for 1045 steel (Fig. 17).

As shown in Figure 17 the obtained results are in good agreement with experimental investigations. This approach has the advantage comparing to classical methods existing in the literature $[8,9]$, to predict the fatigue limit for small defect size. These alone results can be considered as a real improvement of the defect fatigue limit prediction.

\subsubsection{Alternative torsion loading}

In alternative torsion case the affected area is calculated using Equation (14). From an experimental fatigue limit of a medium defect size the parameter $k_{w 2}$ is determined. In this study, a defect size $\sqrt{\text { area }}=400 \mu \mathrm{m}$ corresponding to a fatigue strength $\tau_{D}=145 \mathrm{MPa}$ is used. The assumption 1 gives $k_{w 2}=29700 \mathrm{MPa} . \mu \mathrm{m}$.

In a same graphic the following curves are plotted Figure 18.

- Affected area versus loading amplitude for many defect sizes Equation (14).

- Affected arealimit versus loading amplitude Equation (18).

From the crossing point $\mathrm{N}$ a defect size $\sqrt{\text { area }}=300 \mu \mathrm{m}$ corresponding to fatigue limit $\tau_{D} \approx 156 \mathrm{MPa}$ is deduced. Comparing to experimental results a good accuracy is noted. 


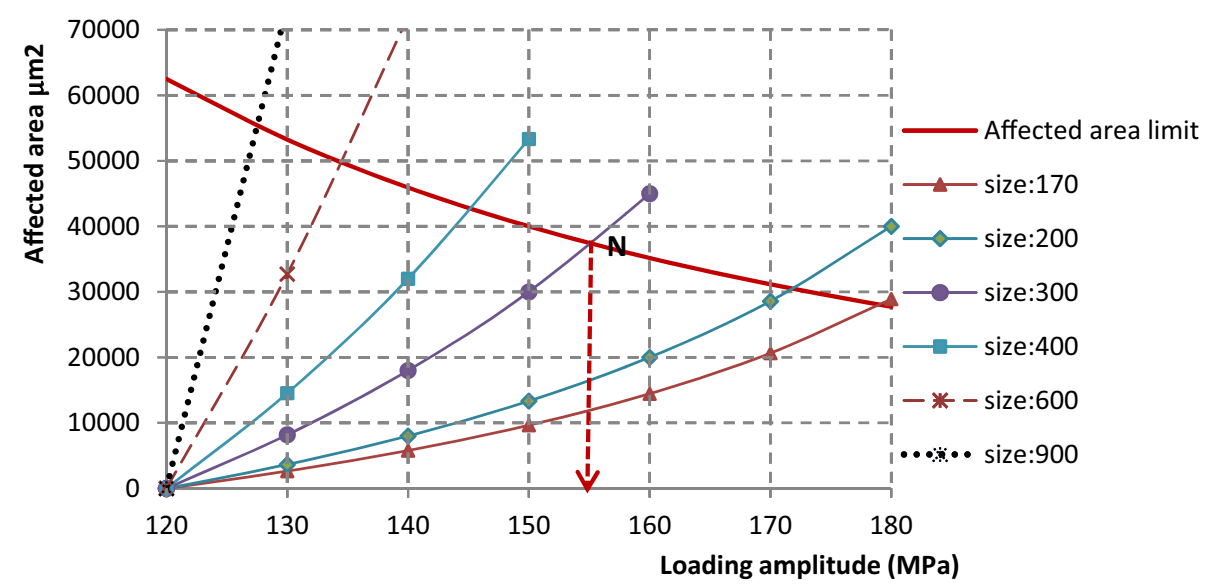

Fig. 18. Application of proposed approach: fully reversed torsion (Material 1045 steel).

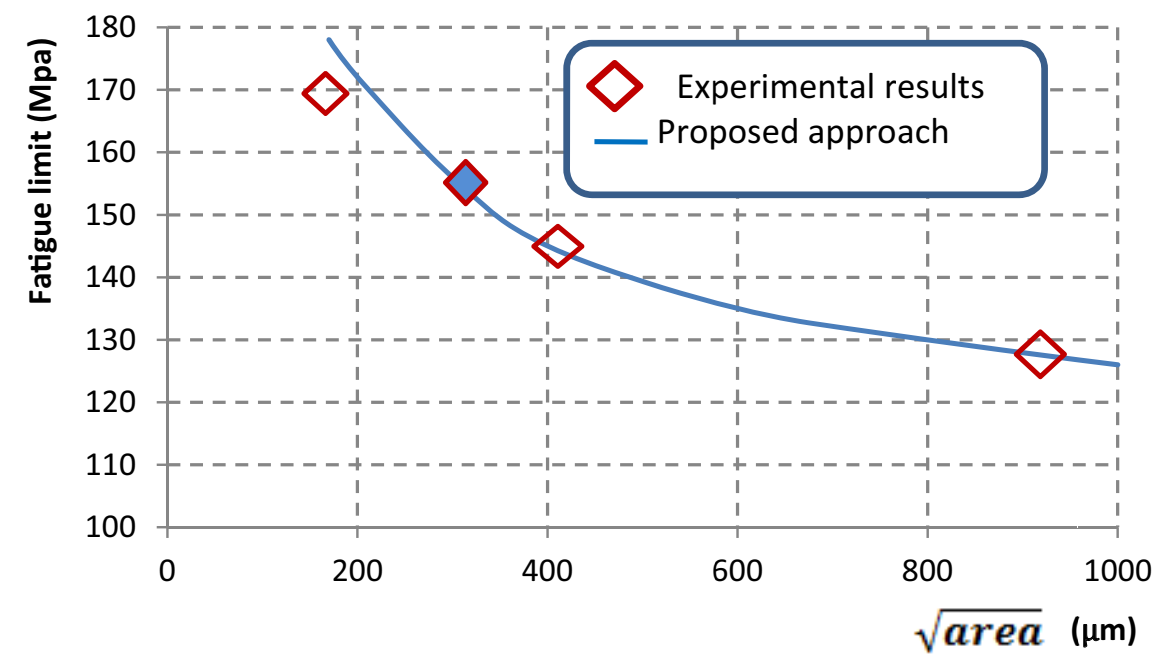

Fig. 19. Kitagawa diagram torsion loading $R_{\sigma}=-1$ (Material 1045 steel).

Using all crossing points, for each defect size Kitagawa diagram can be deduced (Fig. 19) in case of fully reversed torsion loading for 1045 steel.

Obtained results are in good agreement with experimental investigations.

For small defects, the approach is less accurate and over estimates fatigue limit $(\sqrt{\text { area }} \leq 200 \mu \mathrm{m})$.

\subsubsection{Validation for cast aluminium AS7G06-T6 tension loading $R_{\sigma}=-1$}

The used material is a cast aluminium alloy AS7G06T6. Its main mechanical properties are: yield strength $R_{p 0.2}=275 \mathrm{MPa}$, tensile strength $R_{m}=335 \mathrm{MPa}$, Young's modulus $=73 \mathrm{GPa}$ and the Poisson's ratio $\nu=$ 0.3 .

In order to study the evolution of the fatigue limit with defect size, artificial surface defects are produced using the spark erosion principle method. Defects are simplified to spherical void at surface of specimen. A data base for this validation is taken from published results [12].
To apply the proposed approach we need:

- Defect free fatigue limit under fully reversed tension $\sigma_{D-1}$.

- An experimental fatigue limit of defective material with medium defect size.

For cast aluminium defect free the fatigue limit under fully reversed tension is $91 \mathrm{MPa}$. For this material, a defect size $\sqrt{\text { area }}=600 \mu \mathrm{m}$ corresponds to a fatigue strength $\sigma_{D}=85 \mathrm{MPa}$. The use of assumption 1 gives:

$-k_{w 1}=60000 \mathrm{MPa} . \mu \mathrm{m}$. In a same graphic (Fig. 20) the following curves are plotted:

- Affected area versus loading amplitude for many defect sizes Equation (12).

- Affected area limit versus loading amplitude Equation (18).

From the graphic Figure 20 using the proposed methodology explained above we can deduce easily Kitagawa diagram Figure 21.

The proposed approach provides good results for casting aluminium, containing artificial defects, subjected 


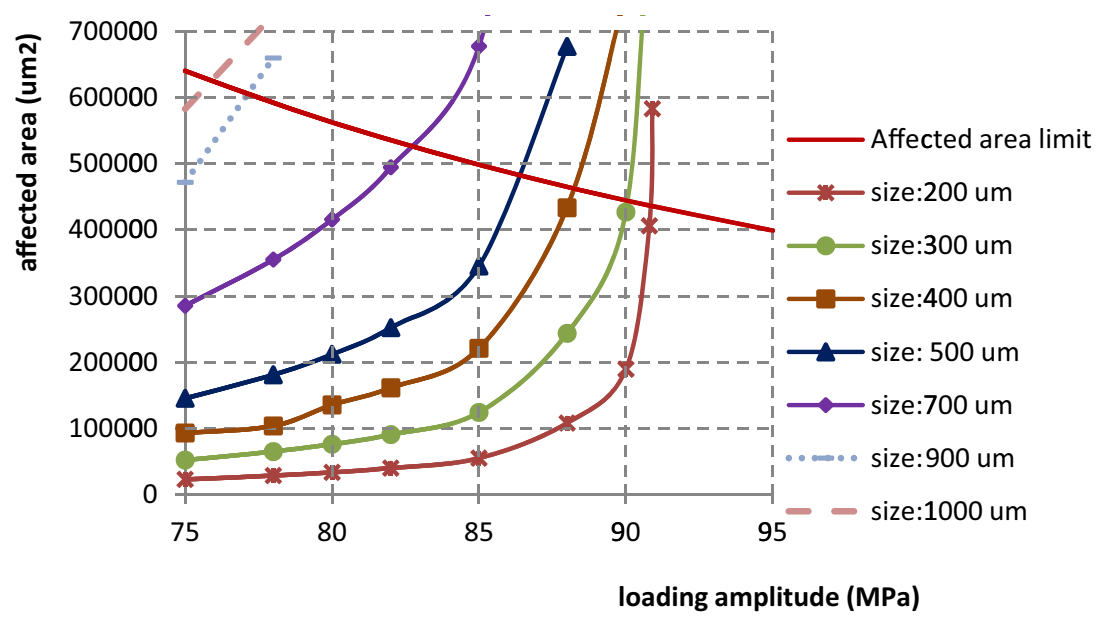

Fig. 20. Application of proposed approach: fully reversed tension (Material AS7G06-T6).

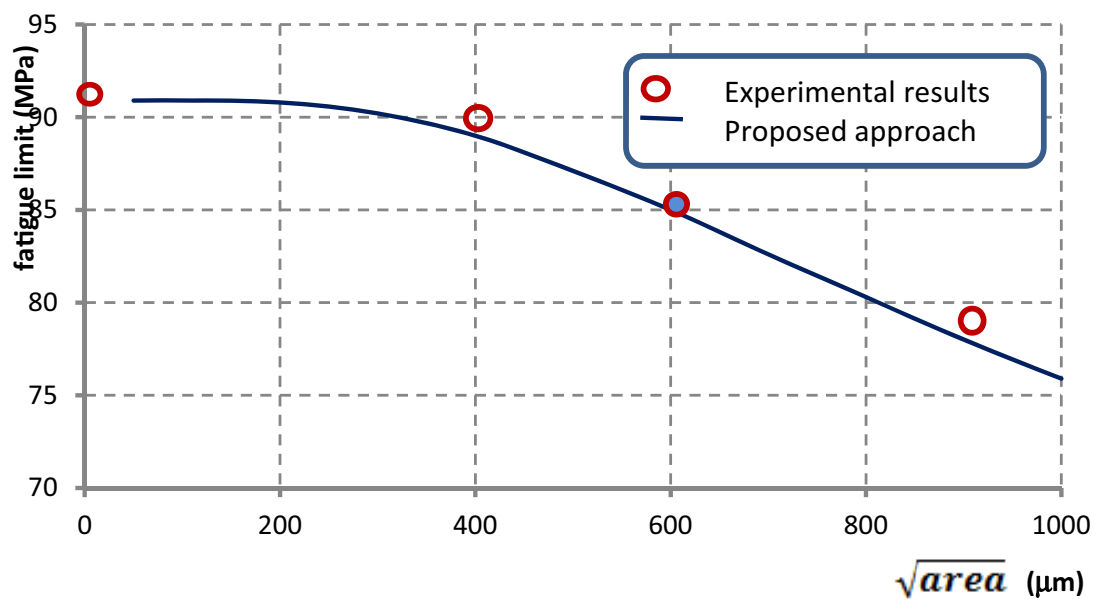

Fig. 21. Kitagawa diagram of tension loading $R_{\sigma}=-1$ (Material AS7G06-T6).

to fully reversed tension. The approach improves that small defect $(\sqrt{\text { area }} \leq 300 \mu \mathrm{m})$ doesn't affect the fatigue limit which is in good agreement with experimental investigations.

\section{Results and discussions}

FE simulations are conducted for: (i) 1045 steel submitted to fully reversed tension and torsion loading, (ii) AS7G6-T6 cast aluminium submitted to fully reversed tension loading. Figures 17, 19 and 21 show that the proposed approach gives good results with an error less than $3 \%$.

Several observations can then be made:

- In HLP the gradient of equivalent stress is fitted by an explicit form, Equations (6) or (7). This representation is more realistic and accurate than the modelisation recently used by Nadot el al. [9] (Fig. 22), where they use the simple average of equivalent stress gradient relative to the maximum equivalent stress in the tip of defect (point A) and the equivalent stress at $\sqrt{\text { area }}$ distance far from the defect (point B).
- The affected area could be a good parameter to predict the fatigue limit of defective material.

- Affected area takes into account the real distribution of equivalent stress on HLP. The macroscopic crack that leads to a failure of the sample propagates in this plane.

- Comparing to previous approaches, this method has the advantage to predict correctly small defect fatigue limit for tension loading.

- The parameters $k_{w 1}$ and $k_{w 2}$ which depend on material and loading conditions, are evaluated from an experimental fatigue limit of a medium size.

\section{Conclusions}

In this work, a new approach to predict the HCF limit for defective material has been developed. Papadopoulos HCF criterion is used to evaluate equivalent stress around defect. Defects are simplified to semi-spherical void at specimen surface. The distribution of Papadoupolos equivalent stress in HLP is studied and interpolated by two explicit equations. 


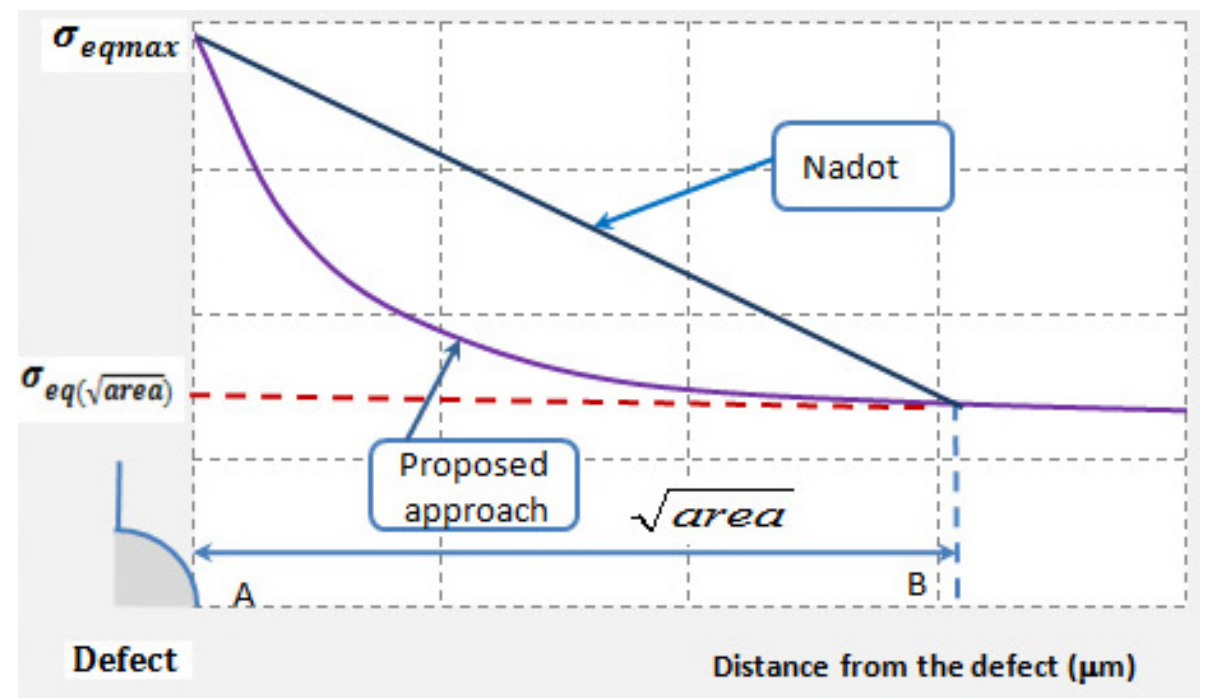

Fig. 22. Comparison between the DSG model and the proposed approach.

A definition of affected area is proposed, from which the Papadopoulos fatigue criterion is violated. The evolution of the affected area, with the amplitude of loading and defect size, allows to determine fatigue limit for different defect sizes.

In this study, the proposed approach is applied to 1045 steel and casting aluminium AS7G06-T6 with introduced defects. The results show a good agreement with the experimental observations. The triaxiality of stress gradient around defect is taken into account by using the affected area in HLP.

The representation of the equivalent stress gradient in HLP by an explicit form, improves fatigue limit prediction comparing to those using the average gradient of hydrostatic pressure or equivalent stress. The proposed approach can be used as an interesting method for engineering design, leading to a more secured HCF behaviour of defective material. This study has to be extended for combined loading with different defect morphology.

\section{References}

[1] Y. Nadot, V. Denier, Fatigue failure of suspension arm: experimental analysis and multiaxial criterion, Eng. Failure Anal. 11 (2004) 485-99

[2] Y. Murakami, M. Endo, The $\sqrt{\text { area }}$ parameter model for small defects and non metallic inclusion in fatigue strength: experimental evidences and application, in Theoretical concepts and numerical analysis of fatigue, edited by A.F. Blom, C.J. Beevers, Birmingham, UK, EMAS, 1992, pp. 51-71

[3] M. Endo, Effects of small defects on the fatigue strength of steel and ductile iron under combined axial/torsional loading. Int Proceeding of third Engineering
Foundation International Conference; Small Fatigue Cracks: Mechanics, Mechanisms and Applications, edited by K.S. Ravichandran, R.O. Ritchie, Y. Murakami, Elsevier, Hawaii, pp. 375-387

[4] Y. Murakami, Metal fatigue: effects of small defects and non metallic inclusion, Oxford, Elsevier, 2002

[5] P. Lukas, L. Kunz, B. Weiss, R. Stickler, Non-damaging notches in fatigue, Fatigue Fract. Eng. Mater. Struct. 9 (1986) 195-204

[6] D. Taylor, Geometrical effects in fatigue: a unifying theoretical model, Int. J. Fatigue 21 (1999) 413-420

[7] Y. Nadot, J. Mendez, N. Ranganathan, A.S. Beranger, Fatigue life assessment of nodular cast iron containing casting defects, Fatigue Fract. Eng. Mater. Struct. 22 (1998) 289-300

[8] T. Billaudeau, Y. Nadot, G. Bezine, Multiaxial fatigue limit for defective materials: mechanisms and experiments, Acta Mater. 52 (2004) 3911-3920

[9] Y. Nadot, T. Billaudeau, Multiaxial fatigue limit criterion for defective materials, Eng. Fracture Mech. (2005)

[10] G. Leopold, Y. Nadot, Fatigue from an induced defect experiments and application of different Multiaxial fatigue approaches, J. ASTM Int. 7 (2010) 1-16

[11] F. Morel, A. Morel, Y. Nadot, Comparison between defects and micro-notches in multiaxial fatigue - the size effect and the gradient effect, Int. J. Fatigue 3 (2009) 263-275

[12] P. Mu, Y. Nadot, C. Nadot-Martin, A. Chabod, I. Serrano-Munoz, C. Verdu, Influence of casting defects on the behaviour of cast aluminium AS7G06-T6, Int. J. Fatigue 63 (2014) 97-109

[13] M. Vincent, C. Nadot-Martin, Y. Nadot, A. Dragon, Fatigue from defect under Multiaxial loading: Defect Stress Gradient (DSG) approach using ellipsoidal equivalent inclusion method, Int. J. Fatigue 9 (2014) 176-187

[14] V. Ioannis, Papadopoulos. Long life fatigue under multiaxial loading, Int. J. Fatigue 23 (2001) 839-849 